\title{
Negligência das classificações diagnósticas atuais com os fenômenos dissociativos do transtorno de estresse pós-traumático
}

\section{Negligence of current diagnostic classifications in dissociative phenomena of posttraumatic stress disorder}

\author{
Alessandra Azevedo Lima ${ }^{1}$, Adriana Fiszman ${ }^{2}$, Carla Marques Portella ${ }^{3}$, Yasmim Andrade Almeida ${ }^{4}$, \\ Fernanda Pereira Salomão ${ }^{5}$, Renata Mendes Guimarães Geoffroy ${ }^{6}$, Ivan Figueira ${ }^{7}$ \\ ${ }^{\dagger}$ Graduada em Psicologia pela Universidade Federal do Rio de Janeiro (UFRJ) e aluna do estágio probatório para mestrado no Instituto de Psiquiatria da UFRJ. \\ ${ }^{2}$ Doutoranda pelo Instituto de Psiquiatria da UFRJ. \\ ${ }^{3}$ Pós-doutoranda do Laboratório de Neurobiologia Il do Instituto de Biofísica Carlos Chagas Filho (CCF) da UFRJ. \\ ${ }^{4}$ Doutorado em Psiquiatria pelo Instituto de Psiquiatria da UFRJ. \\ ${ }^{5}$ Graduanda em Psicologia pela UFRJ. \\ ${ }^{6}$ Graduanda em Psicologia pela UFRJ.
}

7 Professor adjunto do Departamento de Psiquiatria e Saúde Mental da Faculdade de Medicina da UFRJ, coordenador do Laboratório de Transtornos Relacionados ao Estresse do Instituto de Psiquiatria da UFRJ e vice-coordenador do Instituto do Milênio de Violência e Saúde Mental.

Recebido: 20/10/2006 - Aceito: 26/02/2007

\section{Resumo}

Contexto: Apesar da importância crescente da dissociação no transtorno de estresse pós-traumático (TEPT), os sintomas dissociativos não são contemplados pelos critérios diagnósticos das classificações oficiais (CID-10 e DSM-IV). A hipótese de um subtipo dissociativo para o TEPT tem sido corroborada por pesquisas em psicofisiologia, neuroimagem funcional, psicoendocrinologia e sistemas opióides. Pretende-se demonstrar a insuficiência dos critérios vigentes na detecção dos sintomas dissociativos no TEPT mediante relato de um caso com sintomas dissociativos persistentes desde o trauma. Relato de caso: O paciente preenche critérios para TEPT e transtorno depressivo maior pelo DSM-IV. Embora também preencha critérios para transtorno de despersonalização, esse diagnóstico é impossibilitado na presença de TEPT. O paciente apresentou sintomas conversivos peritraumáticos, avaliados pela Escala de Imobilidade Tônica, e sintomas dissociativos proeminentes, peritraumáticos e atuais, já que obteve escores significativos no Questionário de Experiências Dissociativas Peritraumáticas e na Escala de Experiências Dissociativas. Após 3 anos de tratamento, não houve melhora do quadro. Conclusão: O reconhecimento da importância dos fenômenos dissociativos/conversivos no TEPT poderá contribuir para o aprimoramento do diagnóstico, investigações sobre sua fisiopatologia e o desenvolvimento de tratamentos mais eficazes.

Lima, A.A. et al. / Rev. Psiq. Clín. 34 (3); 139-143, 2007

Palavras-chave: TEPT, dissociação, despersonalização, critérios diagnósticos.

\begin{abstract}
Background: In spite of the growing importance of dissociation in post traumatic stress disorder (PTSD), dissociative symptoms are not included in the diagnostic criteria of official classifications (CID-10, DSM-IV). Research in psychophysiology, functional neuroimaging, psycho-endocrinology and opioid systems provides support for a dissociative subtype of PTSD. Case report: A case study on a PTSD patient with persistent dissociative symptoms since the trauma will show that current criteria are insufficient for detecting dissociative symptoms in PTSD. The patient meets criteria for PTSD and major depressive disorder according to the DSM-IV. He also meets criteria for depersonalization disorder, although this diagnosis is not possible with PTSD. The patient showed peritraumatic conversive symptoms, evaluated
\end{abstract}

Endereço para correspondência: Alessandra Azevedo Lima. Rua Álvaro Ramos, 55, casa 5 - CEP 22280-110 - Rio de Janeiro, RJ. E-mail: a_alima@terra.com.br 
by the tonic immobility scale. Further, he obtained significant scores on the Peritraumatic Dissociative Experiences Questionnaire and the Dissociative Experiences Scale, revealing prominent peritraumatic and current dissociative symptoms. There was no improvement in the patient's condition after three years of treatment. Conclusion: Recognition of the importance of dissociative/conversive phenomena in PTSD will allow practitioners to perfect their diagnoses, as well as improve research on the physiopathology of these phenomena and help develop more effective treatments.

Lima, A.A. et al. / Rev. Psiq. Clín. 34 (3); 139-143, 2007

Key-words: PTSD, dissociation, depersonalization, diagnostic criteria.

\section{Introdução}

A atenção aos fenômenos dissociativos associados ao transtorno de estresse pós-traumático (TEPT) vem aumentando desde que este foi, pela primeira vez, introduzido numa classificação psiquiátrica oficial (DSM-III) (American Psychiatric Association, 1980). A dissociação peritraumática é o fator de risco mais estudado para o desenvolvimento desse transtorno (Classen et al., 1998; Spiegel et al., 2000; Ozer et al., 2003) e também tem sido considerada um preditor de gravidade e de má resposta ao tratamento (Lanius et al., 2003; Marshall et al., 1999). Apesar disso, os critérios para TEPT do DSM-IV (American Psychiatric Association, 1994) não enfatizam os fenômenos dissociativos e, por isso, dificultam a sua detecção. Na CID-10 (World Health Organization, 1992), a definição de TEPT é semelhante à do DSM-IV, só não estipula um limite de tempo para o diagnóstico.

A dimensão dissociativa, tão importante na psicopatologia do TEPT, só é contemplada no diagnóstico de transtorno de estresse agudo (TEA) do DSM-IV. Para esse diagnóstico são necessários pelo menos três sintomas dissociativos de um total de cinco. Nas outras dimensões, de revivescência, evitação e hiperestimulação, é necessário apenas um sintoma de cada uma delas, demonstrando a ênfase do TEA na dissociação. Entretanto, o diagnóstico de TEA limita-se ao primeiro mês após o evento traumático e tem sido criticado pela falta de evidências empíricas para validá-lo; sua maior relevância reside no fato de ser um fator de risco para o desenvolvimento de TEPT (Bryant e Harvey, 2000; Lanius et al., 2002; Marshall et al., 1999; Spiegel et al., 2000). Para alguns autores, o TEA não deve, portanto, ser considerado uma entidade nosológica (Bryant e Harvey, 2000; Marshall et al., 1999; Lanius et al., 2002).

Uma alternativa para lidar com a importância da dissociação como parte do diagnóstico de TEPT foi sugerida por Bremner (1999). Ele defendeu a hipótese de dois subtipos diferentes de resposta ao trauma, um com predomínio de intrusão e hiperestimulação e outro com preponderância de sintomas dissociativos. Outros autores também sugeriram que os critérios de TEPT poderiam ser modificados para incluir a dissociação num subtipo mais grave (Marshall et al., 1999).
Os dados empíricos disponíveis para a sustentação de um subtipo dissociativo para o TEPT provêm de estudos de psicofisiologia (Griffin et al., 1997; Koopman et al., 2004; Lanius et al., 2003, 2005), neuroimagem funcional (Lanius et al., 2003, 2005), psicoendocrinologia (Mason et al., 2001; Nuller et al., 2001; Simeon et al., 2003; Stanton et al., 2001) e sistemas opióides (Nishith et al., 2002; Pitman et al., 1990).

Esse relato de caso objetiva ilustrar como aspectos importantes da psicopatologia do TEPT não são adequadamente contemplados pelos sistemas diagnósticos vigentes (DSM-IV e CID-10). O caso clínico foca principalmente a presença de sintomas dissociativos clinicamente relevantes e persistentes no curso do TEPT. Também chama a atenção para as reações agudas apresentadas durante o evento traumático, como os sintomas dissociativos e conversivos.

\section{Descrição do caso clínico}

\section{Avaliação psicométrica do paciente}

Foram utilizados os seguintes instrumentos para o diagnóstico do paciente e a avaliação detalhada de seus sintomas:

- SCID-I (Structured Clinical Interview for DSMIV Axis I Disorders) (Del-Ben et al., 2001) - Os diagnósticos foram obtidos para o relato do caso somente 1 ano após o trauma, quando do início do estudo, por meio do SCID-I, uma entrevista semi-estruturada que fornece os diagnósticos do eixo I pelo DSM-IV. O paciente preencheu os critérios diagnósticos para TEA em avaliação retrospectiva, para TEPT e transtorno depressivo maior, episódio atual, que já estavam sendo tratados desde o sétimo mês após o trauma.

- DES (Dissociative Experiences Scale) (Carlson e Putnam, 1993) - A Escala de Experiências Dissociativas é o questionário mais utilizado mundialmente para o rastreamento e a quantificação de traços dissociativos. A escala é do tipo Likert de 11 pontos, variando de 0 a 10 , e contém 28 itens, com pontuação mínima de 0 e máxima de 100 . O escore de 30 é considerado o ponto de corte 
acima do qual se podem identificar os pacientes com transtornos dissociativos. Ela foi aplicada também no início do estudo, 1 ano após o evento traumático.

- PDEQ (Peritraumatic Dissociative Experiences Questionaire) (Marmar et al., 1998) - O Questionário de Experiências Dissociativas é a escala mais utilizada para quantificar as experiências dissociativas no momento do trauma. Contém 10 itens e é do tipo Likert de 5 pontos, variando de 1 a 5 com pontuação mínima de 10 e máxima de 50. Essa escala peritraumática foi aplicada posteriormente à realização do diagnóstico (SCID-I) e à avaliação dos traços dissociativos (DES). Foi utilizada 2,5 anos após o trauma.

- TIS (Tonic Imobility Scale) (Heidt et al., 2005) - A Escala de Imobilidade Tônica avalia reações de defesa durante o trauma do tipo anestesia, analgesia, congelamento e paralisia. Os sintomas aferidos nessa escala assemelham-se aos conversivos e são considerados a expressão no ser humano da resposta de imobilidade tônica que alguns animais apresentam em situações de perigo inescapável. A TIS contém 12 itens pontuados numa escala de 7 pontos do tipo Likert (de 0 a 6), dos quais 10 são utilizados para a avaliação das reações peritraumáticas. $O$ escore total varia entre 0 e 60 . Os autores da escala subdividiram os 10 itens em dois fatores: imobilidade tônica (IT) e medo. O fator IT é composto por 7 itens (escore de 0 a 42): sensações de congelamento/paralisia, anestesia/analgesia e de frio, incapacidade de se mexer e gritar, certeza de morrer e sensação de desligamento do corpo. O fator medo é composto por 3 itens (escore de 0 a 18): tremer ou se sacudir, sensação de medo e de desligamento do acontecimento. Para considerar a presença de imobilidade tônica peritraumática, exigem-se pontuações iguais ou acima da média, tanto na subescala de IT (média $=21$ ) quanto na subescala de medo (média $=9$ ). Tal como o questionário PDEQ, esta escala peritraumática foi aplicada 2,5 anos após o evento traumático.

\section{Relato do caso}

P. B. é bancário, tem 50 anos, morador do Rio de Janeiro e não tem história de transtorno psiquiátrico anterior ao evento traumático. Sofreu um assalto à mão armada há 3 anos. De acordo com a avaliação psiquiátrica pelo SCID-I, o paciente preencheu os critérios para os seguintes diagnósticos: transtorno de estresse agudo na época do trauma (aferição retrospectiva), transtorno de estresse pós-traumático (TEPT) e transtorno depressivo maior. $\mathrm{O}$ paciente relata que, ao se deslocar de um trabalho para outro, seu carro foi "fechado" por uma perua e uma moto com homens armados com metralhadoras. Quando um dos assaltantes avistou uma patrulha policial, gritou aos outros: "Mata logo ele". Nesse momento, o paciente ficou "paralisado", imaginando que o matariam. Passou as mãos pelo corpo, sentiu como se tivesse levado vários tiros, "como se tivesse morrido" e "como se o espírito tivesse saído do corpo". Permaneceu aterrorizado e tremendo por várias horas. $\mathrm{O}$ paciente referiu diversos sintomas dissociativos, tais como: "perder a noção do que estava acontecendo", "ficar no piloto automático", "assistir ao que acontecia como um espectador", "ter a noção do corpo modificada", "lacunas de memória", "confusão mental" e "desorientação", medidos pelo PDEQ.

Apresentou também sintomas da Escala de Imobilidade Tônica (TIS), como "sentir-se paralisado", "sentir vontade de se mexer, mas ficar travado", "ficar tremendo ou sacudindo", "sentir vontade de gritar, mas ficar travado", "sentir o corpo anestesiado", "sentir medo extremo", "ter certeza de que vai morrer", "sentir-se desligado de si mesmo". O paciente apresentou pontuação máxima em 8 de 10 itens. Foi considerada a ocorrência de imobilidade tônica peritraumática segundo os critérios de Heidt et al. (2005), uma vez que o paciente obteve escore acima da média tanto na subescala de imobilidade tônica (escore $=36$ ) quanto na subescala de medo $($ escore $=13)$.

Em relação ao diagnóstico de TEPT, seus sintomas de revivescência incluem pesadelos que o levam a acordar assustado como se tivesse levado tiros, recordações do trauma como se estivesse acontecendo de novo (flashbacks), medo intenso ao ver veículos semelhantes aos usados durante o evento traumático. Além disso, nos momentos em que se lembra de assuntos relacionados ao trauma, sente o coração acelerar, ficando profundamente angustiado.

Entre os sintomas de hiperestimulação, destacamse comprometimento de concentração e memória e irritabilidade aumentada em relação a barulhos altos de rádio, em lugares onde há muita gente e em viagens de metrô.

Quanto à dimensão de evitação, além de se esquivar do contato com os funcionários da agência em que trabalhava, o paciente também não entra em qualquer outra agência do mesmo banco. Não dirige mais e evita sair à rua. Por muito tempo ficou sem sair de casa e, quando o fazia, sentia-se ansioso. Não vai mais à praia, apesar de gostar muito de caminhar e ouvir o mar. Demonstra também redução generalizada de interesse nas atividades, distanciamento afetivo e sensação de distanciamento em relação a outras pessoas.

O escore da Escala de Sintomas Dissociativos (DES) foi de 31,4, sugerindo a presença de fenômenos dissociativos clinicamente importantes. Desde o trauma, P. B apresenta sintomas dissociativos característicos de despersonalização. Não se sente fazendo parte do seu corpo e estranha a si mesmo ao olhar-se no espelho. Nas atividades do dia-a-dia, não sente coisa alguma, "como se eu não existisse”. Vai automaticamente aos lugares, 
descrevendo que "o corpo vai, mas meu espírito está fora”. Já teve a experiência concreta de ver o próprio corpo andar pela rua.

A desrealização também está presente no relato de que as pessoas, as coisas e o mundo não parecem reais. Diz que passa na rua e não reconhece as pessoas, como se seu mundo não fosse esse. Relata estar aliviado neste "outro mundo para onde foi", "é como um apoio", pois assim acredita estar menos vulnerável. Não lê jornal, nem vê noticiários, evitando tomar conhecimento de fatos violentos.

No início do tratamento, o paciente fez uso de paroxetina $40 \mathrm{mg} / \mathrm{dia}$, sem melhora alguma, principalmente com relação aos sintomas dissociativos. Com cerca de 1 ano de tratamento, a ansiedade diminuiu discretamente, já que o paciente conseguiu ir à rua mesmo angustiado ou temeroso. Após 6 meses com resposta mínima à paroxetina, esta foi substituída por nortriptilina, chegando a $150 \mathrm{mg} / \mathrm{dia}$, mas sem melhora adicional. Atualmente, 3 anos após o evento traumático, faz uso de clonazepan $4 \mathrm{mg} /$ dia, nortriptilina $150 \mathrm{mg} /$ dia, flunitrazepam $30 \mathrm{mg} /$ dia e olanzapina $0,75 \mathrm{mg} /$ dia. No entanto, não houve redução significativa dos sintomas, sobretudo dos dissociativos. Mantêm-se, portanto, os diagnósticos de TEPT e de transtorno depressivo maior. O paciente também vem sendo acompanhado em psicoterapia de base psicanalítica, embora a terapia cognitivo-comportamental seja indicada para o caso, pois o paciente escolheu a abordagem terapêutica de sua preferência.

\section{Discussão}

Este caso ilustra a existência de quadros de TEPT em que há predominância de sintomas dissociativos. Contudo, os fenômenos dissociativos apresentados pelo paciente não foram adequadamente capturados pela seção de TEPT do SCID-I. Já as escalas de dissociação (Escala de Experiências Dissociativas [DES] e Questionário de Experiências Dissociativas Peritraumáticas [PDEQ]) conseguiram detectar esses sintomas. Tais fenômenos dissociativos iniciaram durante e imediatamente após o trauma (medidos pelo PDEQ), tendo sido identificados traços dissociativos clinicamente relevantes (medidos pela DES) que persistiram de forma crônica.

As avaliações das Escalas PDEQ e TIS (Escala de Imobilidade Tônica) estiveram mais sujeitas a possíveis distorções de memória e a interpretações secundárias que o paciente tenha desenvolvido, tais como qualquer avaliação retrospectiva. Mas, por meio delas, percebeu-se que durante o evento traumático o paciente apresentou reações dissociativas e de conversão/ imobilidade tônica, que podem estar relacionadas ao desenvolvimento de TEPT subseqüente. A dissociação peritraumática representa um dos principais fatores de risco para o desenvolvimento do TEPT (Ozer et $a l ., 2003)$. Assim como a dissociação, é possível que a conversão/imobilidade tônica peritraumática constitua um preditor de TEPT uma vez que há uma série de evidências de que a dissociação e a conversão constituem aspectos da mesma condição clínica (Bracha et al., 2005; Nijenhuis et al., 2004; Sar et al., 2004). Com base numa teoria neuroevolutiva, Bracha et al. sugeriram que a conversão está situada ao extremo de um “espectro dissociação/conversão". Heidt et al. (2005) constataram, a partir de uma amostra de 80 mulheres vítimas de abuso sexual na infância, que o relato de imobilidade tônica estava associado a maiores prejuízos psicológicos atuais, em que estavam incluídos TEPT, depressão e ansiedade.

No primeiro mês após o trauma, o paciente preencheu critérios para o transtorno de estresse agudo (TEA), o qual enfatiza os sintomas dissociativos. Desenvolveu, em seguida, TEPT, o que corrobora o caráter preditor da dissociação e do TEA para o TEPT. Após o tratamento ainda estão mantidos os diagnósticos de TEPT e de transtorno depressivo maior, sem melhora dos sintomas dissociativos.

É fundamental indicar a possível correspondência dos sintomas dissociativos peritraumáticos à má resposta ao tratamento, tendo em vista as evidências nesta direção já apresentadas. Nem por isso, no entanto, este estudo pode ter valor conclusivo a respeito do tema, uma vez que se trata de um relato de caso. Ademais, outros tipos de intervenções não utilizadas pelo paciente têm sido recomendados, como as terapias cognitivo-comportamentais e também intervenções psicossociais.

Embora o paciente preencha os critérios diagnósticos para transtorno de despersonalização no DSM-IV, esse diagnóstico não pode ser dado uma vez que o diagnóstico de despersonalização "não pode ocorrer durante o curso de outro transtorno mental" como o TEA e o TEPT (critério D). Na CID-10, o quadro clínico também se adapta à descrição do transtorno de despersonalização/desrealização, entretanto o transtorno não deve ser considerado o diagnóstico principal na presença de outro transtorno. Assim, quadros de TEPT em que há sintomas dissociativos proeminentes acabam mal diagnosticados, com prejuízo no tratamento desses sintomas.

A definição de um subtipo dissociativo para o TEPT, conforme defendido por Bremner (1999), possibilitaria a melhor detecção dos fenômenos dissociativos no TEPT e um tratamento mais específico de pacientes com TEPT e sintomas dissociativos acentuados. Outra alternativa para compensar a negligência aos fenômenos dissociativos nos critérios diagnósticos do TEPT seria permitir o diagnóstico de transtornos dissociativos mesmo na presença do TEPT. O reconhecimento e a valorização da importância dos fenômenos dissociativos/conversivos associados ao TEPT vão permitir o aprimoramento do diagnóstico, a realização de investigações sobre a sua fisiopatologia e o desenvolvimento de estratégias terapêuticas mais eficazes. 


\section{Referências}

American Psychiatric Association. Diagnostic and statistical manual of mental disorders (DSM-III). 3. ed. Washington, DC, 1980.

American Psychiatric Association. Diagnostic and statistical manual of mental disorders. (DSM-IV). 4. ed. Washington, DC, 1994.

Bracha, H.S. et al. Evolution of the human fear-circuitry and acute sociogenic pseudoneurological symptoms: the Neolithic balanced-polymorphism hypothesis. J Affect Disord 88:119-129, 2005.

Bremner, J.D. Acute and chronic responses to psychological trauma: where do we go from here? Am J Psychiatry 156:349-351, 1999.

Bryant, R.A.; Harvey, A.G. New DSM-IV diagnosis of acute stress disorder. Am J Psychiatry 157:1889-1891, 2000.

Carlson, E.B.; Putnam, F.W. An update on the dissociative experiences scale. Dissociation 6:16-26, 1993.

Classen, C. et al. Acute stress disorder as a predictor of posttraumatic stress symptoms. Am J Psychiatry 155:620-624, 1998.

Del-Ben, C. et al. Confiabilidade da entrevista clínica estruturada para o DSM-IV - Versão clínica traduzida para o português. Rev Bras Psiquiatr 23:156-159, 2001.

Griffin, M.G.; Resick, P.A.; Mechanic, M.B. Objective assessment of peritraumatic dissociation: psychophysiological indicators. Am J Psychiatry 154:1081-1088, 1997.

Heidt, J.M.; Marx, B.P.; Forsyth, J.P. Tonic immobility and childhood sexual abuse: a preliminary report evaluating the sequela of rape-induced paralysis. Behav Res Ther 43:1157-1171, 2005.

Koopman, C. et al. Relationships of dissociation and childhood abuse and neglect with heart rate in delinquent adolescents. J Trauma Stress 17:47-54, 2004.

Lanius, R.A.; Hopper, J.W.; Menon, R.S. Individual differences in a husband and wife who developed PTSD after a motor vehicle accident: a functional MRI case study. Am J Psychiatry 160:667-669, 2003.

Lanius, R.A. et al. Brain activation during script-driven imagery induced dissociative responses in PTSD: a functional magnetic resonance imaging investigation. Biol Psychiatry 52:305-311, 2002.
Lanius, R.A. et al. Functional connectivity of dissociative responses in posttraumatic stress disorder: a functional magnetic resonance imaging investigation. Biol Psychiatry 57:873-884, 2005.

Marmar, C.R.; Weiss, D.; Metzler, T. The Peritraumatic Dissociative Experiences Questionnaire. In: Bremner, J.D.; Marmar, C.R., eds. Trauma, memory, and dissociation. 1. ed. American Psychiatric Press, Washington, DC, pp. 249-252, 1998.

Marshall, R.D.; Spitzer, R.; Liebowitz, M.R. Review and critique of the new DSM-IV diagnosis of acute stress disorder. Am J Psychiatry 156:1677$1685,1999$.

Mason, J.W. et al. Psychogenic lowering of urinary cortisol levels linked to increased emotional numbing and a shame-depressive syndrome in combat-related posttraumatic stress disorder. Psychosom Med 63:387-401, 2001.

Nijenhuis, E.R. et al. Somatoform dissociation, reported abuse and animal defence-like reactions. Aust N Z J Psychiatry 38: 678-686, 2004.

Nishith, P.; Griffin, M.G.; Poth, T.L. Stress-induced analgesia: prediction of posttraumatic stress symptoms in battered versus nonbattered women. Biol Psychiatry 51:867-874, 2002.

Nuller, Y.L. et al. Effect of naloxone therapy on depersonalization: a pilot study. J Psychopharmacol 15:93-95, 2001.

Ozer, E.J. et al. Predictors of posttraumatic stress disorder and symptoms in adults: a meta-analysis. Psychol Bull 129:52-73, 2003.

Pitman, R.K. et al. Naloxone-reversible analgesic response to combat-related stimuli in posttraumatic stress disorder. A pilot study. Arch Gen Psychiatry 47:541-544, 1990.

Sar, V. et al. Childhood trauma, dissociation, and psychiatric comorbidity in patients with conversion disorder. Am J Psychiatry 161:2271-2276, 2004.

Simeon, D. et al. Basal norepinephrine in depersonalization disorder. Psychiatry Res 121:93-97, 2003

Spiegel, D.; Classen, C.; Cardena, E. New DSM-IV diagnosis of acute stress disorder. Am J Psychiatry 157:1890-1891, 2000.

Stanton, B.R. et al. Basal activity of the hypothalamic-pituitary-adrenal axis in patients with depersonalization disorder. Psychiatry Res 104:85-89, 2001.

World Health Organization. The CID-10 classification of mental and behavioural disorders: clinical descriptions and diagnostic guidelines. Geneva, The Royal College of Psychiatrists, 1992. 